\title{
Strategyproof Multi-Item Exchange Under Single-Minded Dichotomous Preferences
}

\author{
Haris Aziz
}

\begin{abstract}
We consider multi-item exchange markets in which agents want to receive one of their target bundles of resources. The model encompasses well-studied markets for kidney exchange, lung exchange, and multi-organ exchange. We identify a general and sufficient condition called weak consistency for the exchange mechanisms to be strategyproof even if we impose any kind of distributional, diversity, or exchange cycle constraints. Within the class of weakly consistent and strategyproof mechanisms, we highlight two important ones that satisfy constrained Pareto optimality and strong individual rationality. Several results in the literature follow from our insights. We also derive impossibility results when constrained Pareto optimality is defined with respect to more permissive individual rationality requirements.
\end{abstract}

Keywords Housing markets $\cdot$ Kidney exchange $\cdot$ Organ exchange $\cdot$ Strategyproofness $\cdot$ Pareto optimality $\cdot$ Individual rationality

JEL Classification: C70 - D61 - D71

\section{Introduction}

Clearing complex exchange markets are one of the successful applications of algorithmic economics and multi-agent systems (see e.g., (Sönmez and Ünver, 2011)). Key domains for which algorithms have been designed include housing markets and kidney exchange markets. In recent years, market designers are turning their attention to liver and lung exchange markets. There are proposals to explore the efficiency gains via multi-organ exchange markets (see e.g., (Dickerson and Sandholm, 2017)). Apart from exchanging organs or housing, several new digital platforms have come up that facilitate bartering of goods. Exchange markets are also useful to model timebank scenarios in which people exchange services rather than items.

UNSW Sydney and Data61, CSIRO, Sydney 2052, Australia

Tel.: +61-2-8306 0490

Fax: +61-2-8306 0405

E-mail: haris.aziz@unsw.edu.au 
In most of the organ exchange markets, it is supposed that agents have singleminded dichotomous preferences over outcomes: they are either satisfied with an allocation or they are not. We consider a general model of item or organ exchange that captures all such organ markets. Each agent is endowed with a set of items and each agent has a set of target sets of items. An agent is satisfied if she gets any one of those target sets of items.

The primary focus both in theory and practice is on the optimisation problem of satisfying the maximum number of agents. The problem is computationally complex even for single-unit allocations if there is a bound on the size of the exchange cycles (Abraham et al., 2007). It is also computationally hard if there are agents who have multi-unit demands as is the case of needing two liver or lung components (Luo and Tang, 2015). In this paper, we do not pursue computational issues but focus on the issue of strategyproofness of mechanisms.

In exchange settings, strategyproofness is an important property to ensure the stability and efficiency of a market. Manipulable mechanisms may give incentives for agents to hide their resources which can affect the efficiency of the market. They can also give incentives to agents to misreport their preferences in which case the mechanisms may be optimising on the wrong input. In contrast to traditional voting settings, in which strategyproofness and Pareto optimality be achieved by implementing some variant of serial dictatorship, the same argument need not work for exchange settings in which agents have the ability to 'veto' certain outcomes because individual rationality is imposed.

Our primary contribution is formalizing a general property of mechanisms called weak consistency and proving that any mechanism satisfying weak consistency is strategyproof. Within the class of weakly consistent mechanisms, we highlight two subclasses of mechanisms which have proved useful in restricted domains. The first one is called constrained priority $(C P)$ and the second one is called constrained utilitarian priority $(C U P)$. Both mechanisms run a serial dictatorship type priority mechanism on the set of feasible and individually rational allocations. We show that the mechanisms satisfy strategyproofness and constrained Pareto optimality. We then show how a subtle difference in imposing a different version of individual rationality results in impossibility results even for single-minded agents.

\section{Related Work}

The design of individually rational, Pareto efficient and strategyproof algorithms for exchange problems is one of the most intensely studied topics in market design. Under strict preferences, single-unit demands and single-unit allocations, the wellknown Gale's Top Trading Cycles algorithm satisfies all the three 'gold standard' properties. If the preferences are allowed to be weak, there exist extensions of the TTC algorithm that satisfy the three properties (see e.g., (Jaramillo and Manjunath, 2012: Plaxton, 2013; Saban and Sethuraman, 2013)).

There has also been work on strategyproof exchange with multiple endowments. When preferences are not dichotomous and agents own more than one item, then strategyproofness, Pareto efficiency, and individual rationality are generally impossi- 
ble to achieve (see e.g., (Papai, 2007; Konishi et al., 2001; Todo et al., 2014)). Some positive results depend on the fact that agents have lexicographic preferences over items or items types. Under these assumptions, it is possible to construct extensions of TTC that achieve strategyproofness, individual rationality, and Pareto optimality (see e.g., (Fujita et al., 2015; Sikdar et al., 2017)). The preferences we consider do not assume any of these structural restrictions that are based on lexicographic or conditional lexicographic comparisons. Biró et al. (2015) examined a discrete exchange setting in which agents have strict preferences over objects but there can be multiple copies of objects. They study the conditions under which strategyproofness can be achieved.

There has also been work on multi-item exchanges when preferences are dichotomous. Roth et al. (2005) show that for pairwise kidney exchanges, a priority mechanism is strategyproof. In a highly insightful paper, Hatfield (2005) extended the argument to any kidney exchange framework with any kind of constraints on the feasible allocation including bounds on the exchange cycles. More recently, Luo and Tang (2015) considered lung exchange and again showed that a priority mechanism applied to the set of allocations satisfying the maximum number of agents is strategyproof. Abbassi et al. (2015) considered general exchange problems in which agents partition the items into liked and disliked. Agents' utilities are not single-minded because they are interested in maximizing the number of liked items. For their model, Abbassi et al. (2015) presented an algorithm that is strategyproof and Pareto optimal. However, the algorithm relies on the fact that there are no constraints such as on the size of exchange cycles.

When preferences are single-minded, computing an allocation that satisfies the maximum number of agents is an NP-hard problem even if each agent has two items (Luo and Tang, 2015). Even for kidney exchange in which each agent gets at most one item, computing an allocation that satisfies the maximum number of agents is an NP-hard problem if there is an upper bound on the size of the exchange cycles (Abraham et al., 2007). In this paper, we do not focus on computational problems.

Dichotomous preferences are well-studied in other context as well such as two-sided matching (Bogomolnaia and Moulin, 2004), auctions (Mishra and Roy, 2013), non-cooperative games (Harrenstein et al., 2001), single-winner voting (Brams and Fishburn, 2007), committee voting (Aziz et al., 2017) and probabilistic voting (Bogomolnaia et al., 2005).

\section{Model and Concepts}

An exchange market is a tuple $I=(N, O, e, D)$ where $N=\{1, \ldots, n\}$ be a set of $n$ agents and $O$ be the set of items. The vector $e=\left(e_{1}, \ldots, e_{n}\right)$ specifies the endowment $e_{i} \subset O$ of each agent $i \in N$. We suppose that $\bigcup_{i \in N} e_{i}=O$ and $e_{i} \cap e_{j}=\emptyset$ for all $i, j \in N$ such that $i \neq j$. . Each agent has a demand set $D_{i} \subset 2^{2^{\circ}}$. Each element of $D_{i}$ is a bundle of items that is acceptable to agent $i$ and meets her goal of being in the exchange market. We say that $I^{\prime}=\left(N, O, e^{\prime}, D^{\prime}\right)$ is more constrained than 
$I=(N, O, e, D)$ if $e_{i}^{\prime} \subseteq e_{i}$ for all $i \in N$ and $D_{i}^{\prime} \subseteq D_{i}$ for all $i \in N$. We will write $I^{\prime} \leq I$ if $I^{\prime}$ is more constrained than $I$.

Just like the endowment, any allocation $x=\left(x_{1}, \ldots, x_{n}\right)$ specifies the allocated bundle $x_{i} \subset O$ of each agent $i \in N$. In any allocation $x, x_{i} \cap x_{j}=\emptyset$ for all $i, j \in N$ such that $i \neq j$. Where the context is clear, we refer to the allocation bundle $x_{i}$ as the allocation of agent $i$. We say that allocation $x$ satisfies agent $i$ if $x_{i} \supseteq d$ for some $d \in D_{i}$.

For any two allocations $x_{i}, y_{i} \subseteq O$, one can define the preference relation $\gtrsim_{i}$ of agent $i$ where $x_{i} \gtrsim_{i} y_{i}$ if and only if $y_{i}$ satisfies $i$ implies that $x_{i}$ satisfies $i$. The weak preference relation gives rise to the indifference relation $\sim_{i}$ which holds if $x_{i} \gtrsim_{i} y_{i}$ and $y_{i} \gtrsim_{i} x_{i}$. It also gives rise to the strict part $>_{i}$ of the relation where $x_{i}>_{i} y_{i}$ if $x_{i} \gtrsim_{i} y_{i}$ and $x_{i} \Varangle_{i} y_{i}$.

Example 1 Consider an exchange market in which there are 3 agents. Agent 1 has 4 cars $\left\{c_{1}, c_{2}, c_{3}, c_{4}\right\}$. Agent 2 has a valuable painting $p$. Agent 3 has a sports bike $s$ and a helmet $h$. Agent 1 will only be satisfied if she gets a painting and keeps one of the cars. Agent 2 will only be satisfied if she gets car $c_{1}$ or sports bike $s$ and helmet $h$. Agent 3 will be satisfied if she gets car $c_{4}$. The information can be captured in the form of an exchange market $(N, O, e, D)$ where

- $N=\{1,2,3\}$

- $O=\left\{c_{1}, c_{2}, c_{3}, c_{4}, h, p, s\right\}$

- $e_{1}=\left\{c_{1}, c_{2}, c_{3}, c_{4}\right\} ; e_{2}=\{p\} ; e_{3}=\{s, h\}$

- $D_{1}=\left\{\{c, p\}: c \in e_{1}\right\} ; D_{2}=\left\{\left\{c_{1}\right\},\{s, h\}\right\} ; D_{3}=\left\{c_{4}\right\}$.

A possible allocation that satisfies all the agents is $x$ where $x_{1}=\left\{p, c_{1}, c_{2}, c_{3}\right\} ; x_{2}=$ $\{s, h\} ; x_{3}=\left\{c_{4}\right\}$.

An allocation $x$ is strongly individually rational (S-IR) if $x_{i} \supseteq e_{i}$ or $x_{i} \supseteq d$ for some $d \in D_{i}$. Informally, if an agent's goal is not met, she is not interested in using up any of her endowed resources. This is a standard assumption is settings such as kidney exchange. Except for the last section, we will restrict our attention to S-IR allocations. Enforcing the S-IR requirement can also be seen as enforcing the standard individual rationality requirement for agents that have a special type of trichotomous preferences. In such special trichotomous preferences, agents ideally want to get an acceptable allocation, then they next prefer getting their endowment, and they least prefer a bundle which does not satisfy them and which is not their endowment. Enforcing the S-IR requirement can also be viewed as a special type of feasibility constraint.

Our model can also model altruistic donors who are happy with any allocation. We are interested in maximizing the number of satisfied agents. We say that an allocation $x$ is constrained Pareto optimal within the set of allocations satisfying set $\tau$ of properties if there exists no allocation $y$ satisfying $\tau$ such that $y_{i} \gtrsim_{i} x_{i}$ for all $i \in N$ and $y_{i}>_{i} x_{i}$ for some $i \in N$.

The Generality of the Model Our model captures any kind of exchange market in which agents are interested in getting one of the acceptable bundles. If $\left|e_{i}\right|=1$ for 
each $i \in N$ and $|d|=1$ for each $d \in D_{i}$, the market can model a kidney exchange market (Abraham et al., 2007). If $\left|e_{i}\right|=2$ for each $i \in N$ and $|d|=2$ for each $d \in D_{i}$, the market can model a lung exchange market (Luo and Tang, 2015). It can also model multi-organ exchange market (Dickerson and Sandholm, 2017). The model also allows for altruistic donors who have real items to give but in return, they are happy to get null items. We allow $O$ to contain dummy or null items so as allow imbalanced exchanges in which an agent may get different numbers of items that she gives away. In that case, any allocation for $I=(N, O, e, D)$ has a one-to-one correspondence with a set of cycles in which each item points to her agent and each agent points to an item she gets in the allocation. The cycles are referred to as exchange cycles.

Mechanisms A mechanism $M$ is a function that maps each problem instance $I=$ $(N, O, e, D)$ into an allocation. We say that a mechanism is S-IR if it returns an S-IR allocation for each instance.

Let $\rho(I)$ denote the set of allocations that are feasible with respect to some feasibility constraints denoted by $\rho$. Depending on what the feasibility constraints $\rho$ capture, the term $\rho(I)$ could denote the set of allocations based on pairwise exchanges or exchanges cycles of size at most 3 , or satisfying some distribution or diversity constraint. In principle, $\rho$ could also capture the S-IR requirement. Whatever feasibility conditions we assume on the model, we will assume that they allow the endowment allocation to be a feasible allocation. That is the only minimal assumption we make on the feasibility constraints. It ensures that there always exists at least one allocation satisfying the feasibility constraints.

Since we are interested in mechanisms that satisfy any given feasibility constraints represented by $\rho$, we can view a mechanism as a function that maps the set of $\rho$-feasible allocations to some $\rho$-feasible allocation. Without loss of generality, we can think of mechanisms as choice functions that choose one of the 'best' allocations from the set of feasible allocations. More generally, we will refer to $M(I, Y)$ as forcing $M$ to return an allocation from the set of allocations $Y \subseteq \rho(I)$.

Whereas each agent's private information is $\left(e_{i}, D_{i}\right)$, she can report any $e_{i}^{\prime}$ and $D_{i}^{\prime}$. We will assume $i$ can only declare an endowment that she actually has, therefore any reported endowment $e_{i}$, we assume that $e_{i}^{\prime} \subseteq e_{i}$. We say that a mechanism is strategyproof if there exists no instance under which some agent has an incentive to misreport her private information to get a more preferred outcome for herself. For strategyproof mechanisms, truth-telling is a dominant strategy for each agent.

\section{Weak consistency implies strategyproofness}

We first define weak consistency. Recall that in our setting, for any agent $i$ with demand set $D_{i}$, and for any two allocations $x_{i}$ and $y_{i}$, it is the case that $x_{i} \sim_{i} y_{i}$ if both $x_{i}$ and $y_{i}$ satisfy $i$ with respect to $D_{i}$ or neither satisfy her. 
Consider $\rho(I)$ a set of feasible allocations defined with respect to some feasibility constraints $\rho$. A mechanism $M$ is weakly consistent if for all $I=(N, O, e, D)$,

for any $I^{\prime} \leq I$, for any $Y, Y^{\prime}$ such that $Y^{\prime} \subseteq Y \subseteq \rho(I)$, and $x=M(I, Y)$, if there exists some feasible allocation $y \in Y^{\prime} \subset Y$ under instance $I^{\prime}$ such that $x_{i} \sim_{i} y_{i}$ for all $i \in N$, then $M\left(I^{\prime}, Y^{\prime}\right)=z$ where $z_{i} \sim_{i} x_{i}$ for all $i \in N$.

The preference relation $\sim_{i}$ used in the definition is with respect to instance $I$. Informally, weak consistency requires that if the set of feasible outcomes is more constrained but it is still possible for each agent to get the same satisfaction level, then each agent does get the same satisfaction level from the returned outcome.

Note that weak consistency is weaker than the consistency condition considered by Hatfield (2005). In our setup, consistency can be written as follows. A mechanism $M$ is consistent if for all $I=(N, O, e, D)$,

for any $I^{\prime} \leq I$, for any $Y, Y^{\prime}$ such that $Y^{\prime} \subseteq Y \subseteq \rho(I)$, and $x=M(I, Y)$, if $x$ is a member of $Y^{\prime}$ and it is feasible allocation under $I^{\prime}$, then $M\left(I^{\prime}, Y^{\prime}\right)=x$.

Weak consistency condition is a weaker form of Sen's $\alpha$ condition (Sen, 1971) or Maskin monotonicity (Maskin, 1999).

Theorem 1 Any weakly consistent and S-IR mechanism is strategyproof.

Proof. Consider a weakly consistent mechanism $M$. Suppose that an agent $i$ reports $\left(e_{i}, D_{i}\right)$ and gets an allocation $x_{i}$. If $x_{i} \supseteq d$ for some $d \in D_{i}$, then agent $i$ has no incentive to misreport. Therefore, we suppose for contradiction that $x_{i} \nsupseteq d$ for each $d \in D_{i}$

Suppose that $i$ reports $\left(e_{i}^{\prime}, D_{i}^{\prime}\right)$ such that the resultant allocation is $x^{\prime}$ and $i$ gets an acceptable bundle $b$ according to $D_{i}$. Since we assumed in our setup that $i$ can only declare an endowment that she actually has, therefore $e_{i}^{\prime} \subseteq e_{i}$. We denote the set of resources given by $i$ to the market by $s$. Since $M$ is weakly consistent, if $i$ only reports bundle $b$ as acceptable and only reports $e_{i}^{\prime}$, then the mechanism should still choose the same allocation for agent $i$. Formally, if $i$ reports $e_{i}^{\prime}=s$ and $D_{i}^{\prime}=\{b\}$, then the allocation for $i$ is $b$.

Since $M$ is weakly consistent and S-IR, we can view $M$ as a choice mechanism choosing from the set of feasible and S-IR allocations. It chose an allocation $x^{\prime}$ when $i$ reported $\left(e_{i}^{\prime}, D_{i}^{\prime}\right)$. Now consider $i$ expanding her reported endowment $e_{i}^{\prime}$ to $e_{i} \supseteq e_{i}^{\prime}$ and her reported demand set $D_{i}^{\prime}$ to $D_{i} \supseteq D_{i}^{\prime}$. When $i$ reports $\left(e_{i}, D_{i}\right)$, some additional feasible and S-IR allocations may emerge as well. When $i$ reports $\left(e_{i}, D_{i}\right)$, the only new feasible S-IR allocations are those in which $i$ gets one of her acceptable bundles according to $D_{i}$. By weak consistency, one of the three cases hold:

(i) the same allocation $x$ is returned in which case $i$ is satisfied with respect to $D_{i}$

(ii) some other allocation $y \neq x$ is returned such that all agents are indifferent with $x$. In this case, $i$ is satisfied with respect to $D_{i}$.

(iii) some allocation $z$ is returned such that some agent is not indifferent between $x$ and $z$. In this case the allocation must be one of the new allocations resulted from $i$ expanding her reported endowment $e_{i}^{\prime}$ to $e_{i} \supseteq e_{i}^{\prime}$ and her reported demand set $D_{i}^{\prime}$ to $D_{i} \supseteq D_{i}^{\prime}$. Such a new allocation cannot be one in which $i$ does not trade 
any item because in that case, it would even be feasible, when $i$ did not expand her reported sets $e_{i}^{\prime}$ and $D_{i}$. Therefore, it must be the case that $i$ did trade some items. Since we assume the S-IR requirement, if $i$ did trade, then $i$ gets some allocation that satisfies her with respect to one of her demands in $D_{i}$.

Since $i$ is satisfied with respect to $D_{i}$ in all the cases, it contradicts the assumption that $i$ benefited from misreporting $\left(e_{i}^{\prime}, D_{i}^{\prime}\right)$.

Our proof technique is along the same lines as Theorem 2 of Hatfield (2005) who proved that for exchange markets with dichotomous preferences and single-unit demands and endowments, any consistent mechanism is strategyproof. In contrast to the result by Hatfield (2005), (1) our result applies to any exchange market where agents can have any number of items (2) it considers a property which is weaker than the consistency property used by him and (3) it explicitly uses the S-IR requirement which was implicitly used in the proof by Hatfield. As a corollary, we recover the central result from the paper by Hatfield. Since we focus on the S-IR requirement explicitly, it leads to a better understanding when the more permissive requirement of IR is used.

\section{Constrained Priority Mechanisms}

In this section, we focus on two natural weakly consistent mechanisms. The mechanisms are adaptations of the idea of applying serial dictatorship and priority over the set of all feasible outcomes (see e.g. (Aziz et al., 2013)). The mechanisms are parametrized with respect to $\rho$ (a set of feasibility constraints) and $\pi$ which is a priority ordering over the agent set in which the agent in $j$-th turn is denoted by $\pi(j)$. Both rules are based on lexicographic comparisons.

For any permutation $\pi$ of $\mathrm{N}$, the CP mechanism is defined as follows.

$$
C P(I, \rho, \pi)=\underset{x \in \rho(I)}{\arg \operatorname{lex} \max }\left(u_{\pi(1)}(x), \ldots, u_{\pi(n)}(x)\right) .
$$

For any permutation $\pi$ of N, the CUP mechanism is defined as follows.

$$
C U P(I, \rho, \pi)=\underset{x \in \rho(I)}{\arg \operatorname{lex} \max }\left(\sum_{i \in N} u_{i}(x), u_{\pi(1)}(x), \ldots, u_{\pi(n)}(x)\right) .
$$

$\mathrm{CP}$ starts from the set of feasible allocations and then refines this set by using a priority ordering over the agents. CUP starts from the set of feasible allocations satisfying the maximum number of agents and then refines this set by using a priority ordering over the agents. Both CP and CUP are flexible enough to enforce or not enforce S-IR as part of the feasibility constraints.

The following remark points out that CUP is a general mechanism that has been used in restricted domains when S-IR is enforced.

Remark 1 When applied to pairwise kidney allocation, CP and CUP are equivalent to the priority mechanism considered by Roth et al. (2005). When applied to kidney exchange, CUP is equivalent to the priority mechanism considered by Hatfield (2005). 
When applied to kidney exchange, CUP is equivalent to the lexicographic mechanism considered by Luo and Tang (2015). When applied to house allocation with existing tenants, CUP is equivalent to the MIR algorithm of Aziz (2019).

Next, it is shown that CUP and CP are weakly consistent.

Lemma $1 C U P$ and $C P$ are weakly consistent.

Proof. For any set of feasible allocations, CP and CUP can be seen as choice functions that choose the 'best' allocation from the set. When that allocation is from removed the set, the next choices of CP and CUP can be selected. Hence both CP and CUP can be seen as inducing a weak order on the set of feasible allocations. In such a weak order, any two feasible allocations in which each agent gets the same utility, are in the same indifference class. If an allocation is removed from the set of feasible allocations, then note that the relative ordering of the other allocations does not change. Hence CP and CUP both satisfy weak consistency.

Theorem 2 For any feasibility restriction $\rho$ on the set of S-IR allocations, CUP is strategyproof.

Proof. The statement follows from Theorem 1 and Lemma1,

Theorem 3 For any feasibility restriction $\rho$ on the set of S-IR allocations, CP is strategyproof.

Proof. The statement follows from Theorem 1 and Lemma1

As corollaries of the above theorem, we obtain several results where the mechanisms referred to in the corollaries are essentially applying $\mathrm{CP}$ to a restricted domain of exchange problems where S-IR is enforced.

Corollary 1 (Theorem 1, Roth et al. (2005)) For pairwise kidney exchange markets, the priority mechanism is strategyproof.

Proof. The statement follows from Theorem 2 and Remark 1.

Corollary 2 (Corollary 3, Hatfield (2005)) For kidney exchange markets, the priority mechanism is strategyproof even if we impose any constraint on the exchange cycle sizes.

Proof. The statement follows from Theorem 2 and Remark 1 .

Corollary 3 (Theorem 2, Luo and Tang (2015)) For lung exchange markets where no cycle constraints are imposed, the lexicographic mechanism (Luo and Tang, 2015) is strategyproof.

Proof. The statement follows from Theorem 2 and Remark 1.

Corollary 4 (Proposition 5, Aziz (2019)) For house allocation with existing tenants, the MIR algorithm (Aziz, 2019) is strategyproof. 
Proof. The statement follows from Theorem 2 and Remark1.

If we include S-IR in the set of feasibility requirements $\rho$, then note that both $\mathrm{CP}$ and CUP return an allocation that is Pareto optimal allocation within the set of allocations satisfying S-IR and $\rho$. Hence, we can rephrase our result in the form of the following theorem.

Theorem 4 Under dichotomous preferences, for any restriction on allocations $\rho$, there exists a strategyproof mechanism that returns an allocation that satisfies constrained Pareto optimality among the set of all allocations that satisfy $\rho$ and S-IR.

Since imposing S-IR is similar to imposing IR when agents have trichotomous preferences, we can rephrase the theorem above as follows.

Theorem 5 Under trichotomous preferences where each agent only has her own endowment as the second preferred outcome, for any restriction on allocations $\rho$, there exists a strategyproof mechanism that returns an allocation that satisfies constrained Pareto optimality among the set of all allocations that satisfy $\rho$ and IR.

\section{Impossibility Results}

In the previous sections, we limited our attention to allocations that satisfy S-IR (strong individual rationality). We now explore the consequence of dropping the S-IR requirement. An allocation $x$ is individually rational (IR) if $e_{i} \supseteq d$ for some $d \in D_{i}$ implies that $x_{i} \supseteq d^{\prime}$ for some $d^{\prime} \in D_{i}$.

Whereas IR is a less stringent requirement than S-IR, constrained Pareto optimality with respect to allocations satisfying IR is a stronger property than constrained Pareto optimality with respect to allocations satisfying S-IR. In fact, the property is stronger enough that our central results in the previous sections collapse. In particular Theorem 5 changes into an impossibility result as we expand the set of feasible allocations.

The proof is based on an adaptation of an impossibility result for strategyproof mechanisms maximizing the total utility of agents in which agents want to get as many desirable items as possible and their utilities are not single-minded (Theorem 1, (Abbassi et al., 2015)). We adapt the argument to only consider agents having singleminded utilities: they are only satisfied if they get one of their targets sets of items. The subtle difference from the previous section is that we do not enforce S-IR which expands the set of feasible of allocations and leads to an impossibility result.

We say that an allocation is a result of pairwise exchanges if it is a result of pairs of agents make one-for-one exchange for items and with each item changing ownership at most once. Considering the exchange cycles view of allocations as mentioned in Section 3, an allocation as a result of pairwise exchange is characterized by exchange cycles in which each cycle has at most two agents in it.

Theorem 6 Consider $\rho$ as the restriction of allowing allocations that are a result of pairwise item exchanges in which agents get desirable items. For $|N| \geq 3$, there exists no strategyproof mechanism that returns an allocation that is constrained Pareto optimal among the set of all allocations that satisfy $\rho$. 
Proof. Consider a set of three agents $N=\{1,2,3\}$.

- $e_{1}=\left\{a_{1}, a_{2}, a_{3}\right\}$.

$-e_{2}=\left\{b_{1}, b_{2}, b_{3}\right\}$.

$-e_{3}=\left\{c_{1}, c_{2}, c_{3}\right\}$.

Each agent $i$ likes some items $A_{i} \subseteq O$ :

- $A_{1}=\left\{b_{1}, b_{3}, c_{1}, c_{2}, c_{3}\right\}$.

- $A_{2}=\left\{a_{1}, a_{3}, c_{1}, c_{2}, c_{3}\right\}$.

- $A_{3}=\left\{a_{2}, a_{3}, b_{2}, b_{3}\right\}$.

- $D_{1}=\left\{S \subset O:|S|=3\right.$ and $\left.S \subset A_{1}\right\}$.

- $D_{2}=\left\{S \subset O:|S|=3\right.$ and $\left.S \subset A_{2}\right\}$.

- $D_{3}=\left\{S \subset O:|S|=3\right.$ and $\left.S \subset A_{3}\right\}$.

Since we allow allocations as a result of pairwise exchanges, the resultant allocation has a corresponding matching $M$ on the node set $O$ that indicates which items are pairwise exchanged. Also, note that each agent owns items that she does not like. Each agent needs three liked items to be satisfied. Since we allow allocations as a result of pairwise exchanges, a satisfied agent need to pairwise exchange all her items with liked items.

We first prove that in any allocation that satisfies $\rho$ and is Pareto optimal, exactly two agents are satisfied. We first prove that at most two agents can be satisfied. Since only allocations as a result of pairwise exchanges are allowed, the maximum sized matching for 9 item nodes is at most 8 . In other words, at most 8 items are exchanged so one agent still keeps an unliked item. Thus, at least one agent can get at most two acceptable items so it is not satisfied. It is also easy to see that at least two agents can be satisfied by pairwise exchanges.

Suppose agent 1 is not satisfied. Then agent 1 can report $A_{1}^{\prime}=\left\{b_{3}, c_{1}, c_{2}, c_{3}\right\}$ and $D_{1}^{\prime}=\left\{S \subset O:|S|=3\right.$ and $\left.S \subset A_{1}^{\prime}\right\}$. In that case, agent 1 is not willing to get item $b_{1}$ in any exchange and can force this because of the requirement we have in $\rho$. Agent 2 has one item which is useless to other agents so in any $\rho$-feasible allocation it cannot be satisfied because it does not get three items from $A_{2}$. Therefore the only constrained Pareto optimal allocations are those in which agent 1 exchanges $a_{1}$ with $b_{3}$, and $a_{2}$ and $a_{3}$ with two the items among $e_{3}$. Therefore by changing her preference relation, agent 1 gets satisfied.

Suppose agent 2 is not satisfied. Then agent 1 can report $A_{2}^{\prime}=\left\{a_{3}, c_{1}, c_{2}, c_{3}\right\}$ and $D_{2}^{\prime}=\left\{S \subset O:|S|=3\right.$ and $\left.S \subset A_{2}^{\prime}\right\}$. In that case, agent 2 is not willing to get item $a_{1}$ in any exchange and can force this because of the requirement we have in $\rho$. Agent 1 has one item which is useless to other agents so it in any $\rho$-feasible allocation it cannot be satisfied because it does not get three items from $A_{1}$. Therefore the only constrained Pareto optimal allocations are those in which agent 2 exchanges $b_{1}$ with $a_{3}$, and $b_{2}$ and $b_{3}$ with two the items among $e_{3}$. Therefore by changing her preference relation, agent 2 gets satisfied.

Suppose agent 3 is not satisfied. Then agent 3 can report $A_{3}^{\prime}=\left\{a_{3}, b_{2}, b_{3}\right\}$ and $D_{1}^{\prime}=\left\{S \subset O:|S|=3\right.$ and $\left.S \subset A_{3}^{\prime}\right\}$. In that case, agent 3 is not willing to get item $a_{2}$ in any exchange and can force this because of the requirement we have in $\rho$. Agent 
1 has one item which is useless to other agents so it in any $\rho$-feasible allocation it cannot be satisfied because it does not get three items from $A_{2}$. Therefore the only constrained Pareto optimal allocations are those in which agent 3 exchanges all her items with the items in the set $A_{3}^{\prime}$. Therefore by changing her preference relation, agent 3 gets satisfied.

The impossibility result is tight in the sense that for $|N| \leq 2$, the properties can be easily satisfied by the $\mathrm{CP}$ mechanism with the constraint of individual rationality. Next, we note that Theorem 6 can be rephrased as follows.

Theorem 7 Consider $\rho$ as the restriction of allowing allocations that are a result of pairwise item exchanges in which agents get desirable items. For $|N| \geq 3$, there exists no strategyproof mechanism that returns an allocation that is constrained Pareto optimal among the set of all allocations that satisfy $\rho$ and IR.

Proof. Consider the proof of the previous theorem. Since none of the agents is satisfied by their endowment, the IR requirement has no bite. In particular, the set of allocations satisfying IR coincides with the set of all allocations. Hence, constrained Pareto optimality among the set of all allocations that satisfy $\rho$ is equivalent to constrained Pareto optimal among the set of all allocations that satisfy $\rho$ and IR.

\section{Conclusions}

We have provided a clearer understanding of strategyproof multi-item exchange markets in which agents have dichotomous preferences. We focussed on scenarios where agents have single-minded preferences: they are either satisfied if they get a target bundle or they are dissatisfied if they do not get a target bundle. Under these preferences, we showed that strategyproofness can be achieved even under arbitrary restrictions on feasible allocations. In fact, there exist mechanisms that satisfy constrained Pareto optimality. We complement these results with a couple of impossibility results when we strengthen constrained Pareto optimality by enforcing a weaker notion of individual rationality. We envisage further algorithmic and game-theoretic research on the general multi-item exchange model discussed in the paper.

\section{Acknowledgments}

Aziz gratefully acknowledges the UNSW Scientia Fellowship and Defence Science and Technology (DST). He thanks Barton Lee and Bahar Rastegari for useful feedback.

\section{References}

Z. Abbassi, N. Haghpanah, and V. Mirrokni. Exchange market mechanisms without money. Technical report, http://people.csail.mit.edu/nima/papers/exchanges.pdf 2015 . 
D. Abraham, A. Blum, and T. Sandholm. Clearing algorithms for barter exchange markets: Enabling nationwide kidney exchanges. In Proceedings of the 8th ACM Conference on Electronic Commerce (ACM-EC), pages 295-304. ACM Press, 2007.

H. Aziz. Mechanisms for house allocation with existing tenants under dichotomous preferences. Journal of Mechanism and Institution Design, 2019. Forthcoming.

H. Aziz, F. Brandt, and M. Brill. The computational complexity of random serial dictatorship. Economics Letters, 121(3):341-345, 2013.

H. Aziz, M. Brill, V. Conitzer, E. Elkind, R. Freeman, and T. Walsh. Justified representation in approval-based committee voting. Social Choice and Welfare, 48(2): 461-485, 2017.

P. Biró, F. Klijn, and S. Papai. Circulation under responsive preferences. 2015.

A. Bogomolnaia and H. Moulin. Random matching under dichotomous preferences. Econometrica, 72(1):257-279, 2004.

A. Bogomolnaia, H. Moulin, and R. Stong. Collective choice under dichotomous preferences. Journal of Economic Theory, 122(2):165-184, 2005.

S. J. Brams and P. C. Fishburn. Approval Voting. Springer-Verlag, 2nd edition, 2007.

J. P. Dickerson and T. Sandholm. Multi-organ exchange. J. Artif. Intell. Res., 60: 639-679, 2017.

E. Fujita, J. Lesca, A. Sonoda, T. Todo, and M. Yokoo. A complexity approach for core-selecting exchange with multiple indivisible goods under lexicographic preferences. In Proceedings of the 29th AAAI Conference on Artificial Intelligence (AAAI), pages 907-913. AAAI Press, 2015.

P. Harrenstein, W. van der Hoek, J.-J. Meyer, and C. Witteveen. Boolean games. In J. van Benthem, editor, Proceedings of the 8th Conference on Theoretical Aspects of Rationality and Knowledge (TARK), pages 287-298, 2001.

J. W. Hatfield. Pairwise kidney exchange: Comment. Journal of Economic Theory, 125:189-193, 2005.

P. Jaramillo and V. Manjunath. The difference indifference makes in strategy-proof allocation of objects. Journal of Economic Theory, 147(5):1913-1946, September 2012.

H. Konishi, T. Quint, and J. Wako. On the Shapley-Scarf economy: the case of multiple types of indivisible goods. Journal of Mathematical Economics, 35(1):1-15, 2001.

S. Luo and P. Tang. Mechanism design and implementation for lung exchange. In Proceedings of the 23rd International Joint Conference on Artificial Intelligence (IJCAI), pages 209-215. AAAI Press, 2015.

E. Maskin. Nash equilibrium and welfare optimality. Review of Economic Studies, 66(26):23-38, 1999.

D. Mishra and S. Roy. Implementation in multidimensional dichotomous domains. Theoretical Economics, 8(2):431-466, 2013.

S. Papai. Exchange in a general market with indivisible goods. Journal of Economic Theory, 132:208-235, 2007.

C. G. Plaxton. A simple family of top trading cycles mechanisms for housing markets with indifferences. In Proceedings of the 24th International Conference on Game Theory, 2013. 
A. E. Roth, T. Sönmez, and M. U. Ünver. Pairwise kidney exchange. Journal of Economic Theory, 125:151-188, 2005.

D. Saban and J. Sethuraman. House allocation with indifferences: a generalization and a unified view. In Proceedings of the 14th ACM Conference on Electronic Commerce (ACM-EC), pages 803-820. ACM Press, 2013.

A. K. Sen. Choice functions and revealed preference. Review of Economic Studies, 38(3):307-317, 1971.

S. Sikdar, S. Adali, and L. Xia. Mechanism design for multi-type housing markets. In Proceedings of the 31st AAAI Conference on Artificial Intelligence (AAAI), pages 684-690, 2017.

T. Sönmez and M. U. Ünver. Matching, allocation, and exchange of discrete resources. In J. Benhabib, M. O. Jackson, and A. Bisin, editors, Handbook of Social Economics, volume 1, chapter 17, pages 781-852. Elsevier, 2011.

T. Todo, H. Sun, and M. Yokoo. Strategyproof exchange with multiple private endowments. In Proceedings of the 28th AAAI Conference on Artificial Intelligence (AAAI), pages 805-811. AAAI Press, 2014. 